\title{
Article \\ Comparative Analysis of the Differences in Dentofacial Morphology According to the Tongue and Lip Pressure
}

\author{
Yoo-Sun Lee ${ }^{1}$, Jiho Ryu ${ }^{1}$, Seung-Hak Baek ${ }^{1} \mathbb{D}$, Won Hee Lim ${ }^{1}$, Il-Hyung Yang ${ }^{1}$, Tae-Woo Kim ${ }^{1, *(\mathbb{D})}$ \\ and Seok-Ki Jung ${ }^{2, * \mathbb{D}}$ \\ 1 Department of Orthodontics, School of Dentistry, Dental Research Institute, Seoul National University, \\ Seoul 03080, Korea; yousunzzang@snu.ac.kr (Y.-S.L.); jiho@snu.ac.kr (J.R.); drwhite@snu.ac.kr (S.-H.B.); \\ whlim@snu.ac.kr (W.H.L.); drortho@snu.ac.kr (I.-H.Y.) \\ 2 Department of Orthodontics, Korea University Ansan Hospital, Ansan, Gyeonggi 15355, Korea \\ * Correspondence: taewoo@snu.ac.kr (T.-W.K.); jgosggg@korea.ac.kr (S.-K.J.)
}

check for

updates

Citation: Lee, Y.-S.; Ryu, J.; Baek, S.-H.; Lim, W.H.; Yang, I.-H.; Kim,

T.-W.; Jung, S.-K. Comparative

Analysis of the Differences in

Dentofacial Morphology According to the Tongue and Lip Pressure.

Diagnostics 2021, 11, 503. https://

doi.org/10.3390/diagnostics11030503

Academic Editor: Natalia

D. Gladkova

Received: 4 February 2021

Accepted: 10 March 2021

Published: 12 March 2021

Publisher's Note: MDPI stays neutral with regard to jurisdictional claims in published maps and institutional affiliations.

Copyright: (C) 2021 by the authors. Licensee MDPI, Basel, Switzerland. This article is an open access article distributed under the terms and conditions of the Creative Commons Attribution (CC BY) license (https:// creativecommons.org/licenses/by/ $4.0 /)$.

\begin{abstract}
The aim of this study was to evaluate the effects of the tongue and lip pressure on dentofacial morphology. The subjects comprised 194 patients with malocclusion. Anterior and posterior tongue elevation and lip pressures were evaluated using the Iowa Oral Performance Instrument (IOPI) device. The lateral cephalograms of each subject were traced and digitized to perform the analysis. Statistical analysis was used to investigate the relationship between perioral muscle force and the cephalometric variables. Anterior and posterior tongue pressure was both higher in males than in females. No sex difference in lip pressure was observed. The group with a low posterior tongue pressure showed a short ramus height, short posterior facial height, and clockwise-rotated mandible. On the other hand, lip pressure had a significant influence on maxillary incisor angulation. Skeletal pattern was not found to be significantly related with lip pressure. The anterior tongue pressure appeared as a mixed pattern of the two results. Tongue pressure was related to skeletal measurements, such as short posterior facial height, and lip pressure was related to the angulation of the anterior teeth. This study suggests that there may be differences in dentofacial morphology according to the differences in perioral muscle force.
\end{abstract}

Keywords: tongue pressure; lip pressure; dentofacial morphology; iowa oral performance instrument

\section{Introduction}

The balance between the tongue and perioral muscle is known to affect the position of the teeth, and it plays a significant role in the formation and maintenance of the dental arch form [1-4]. Due to the fact that the teeth are positioned between the lips, cheeks, and tongue, the force from this muscle is the major factor that determines the tooth position. It is important to evaluate the dynamics of the perioral muscle at rest and during function, as the function of the perioral muscle is closely related to the onset of malocclusion [5-7]. In addition, the contribution of the strength of the lips, cheeks and tongue is important to orthodontists for proper treatment planning and achieving posttreatment stability [8].

The tongue is a powerful muscular organ that exerts pressure at frequent intervals during the day and night, performing various functions, such as mastication, swallowing, and phonation. Several studies have reported the effect of tongue size on the dental arch $[4,9,10]$. Proffit stated that the resting position of the tongue is among the key factors in the maintenance of the dental equilibrium [11]. It has been shown that the tongue posture is lower in skeletal class III patients than in class I patients [3].

However, previous studies regarding the effect of tongue pressure on the dentofacial morphology have shown different results. Kurabeishi et al. showed that maximum tongue pressure is significantly lower in the skeletal class II group than in the skeletal class III group [12]. On the other hand, several studies have suggested that tongue pressure is not related to the craniofacial morphology $[13,14]$. The limitations of these studies were 
that they measured only the anterior pressure of the tongue tip, and only the anteroposterior skeletal difference was evaluated. Despite the many previous studies on tongue pressure, the relationship between dentofacial morphology and tongue pressure remains uncertain $[13,15,16]$.

Some studies have been conducted to understand the effect of lip closure force on the onset of malocclusion [14,17]. Jung et al. showed that the upper incisor inclination is related to the lip-closing force in males with normal molar occlusion [18]. Other studies have reported that the lip-closing force produced by subjects of angle class II division 1 are weaker than those of angle class I relationships [2].

However, in previous studies, a self-made measuring device or a modified sensor has been used to measure the pressure at a specific intraoral location. Due the fact that the measurement methods are not standardized, results cannot be compared.

Therefore, the aim of this study was to evaluate the effects of the tongue and lip pressure on dentofacial characteristics using a standardized measuring device. Since the contribution of tongue and lip pressure is an important part in the treatment planning, the significance of this study can be found.

\section{Materials and Methods}

\subsection{Subjects}

The sample consisted of 194 patients who visited the department of orthodontics at Seoul National University Dental Hospital. At the initial orthodontic consultation, an informed consent was obtained from the patients. Patients with maxillofacial deformities and neurological disorders were excluded. The sample included 104 men and 90 women (mean age: 23.65 ; SD: 7.3 ).

\subsection{Measurement Methods of the Lateral Cephalometric Radiographs}

Cephalometric radiographs were taken with the CX-90SP cephalostat (Asahi Co, Kawasaki, Japan), 72 to $74 \mathrm{k}$ (peak), $20 \mathrm{~mA} / \mathrm{sec}$, at the Department of Oral Maxillofacial Radiology in Seoul National University Dental Hospital. Each radiograph was taken in the natural head position. The natural head position is a reproducible position of the head in an upright posture, and the eyes look at the point of the eye level position. In addition, all patients were instructed to hold their breath and not swallow while the radiographs were being taken.

A single investigator performed all the tracings. The reference points were digitized with V-ceph (ver 5.3, Osstem Inc., Seoul, Korea). Twenty landmarks and 28 measurements were used in this study (Figure 1). 

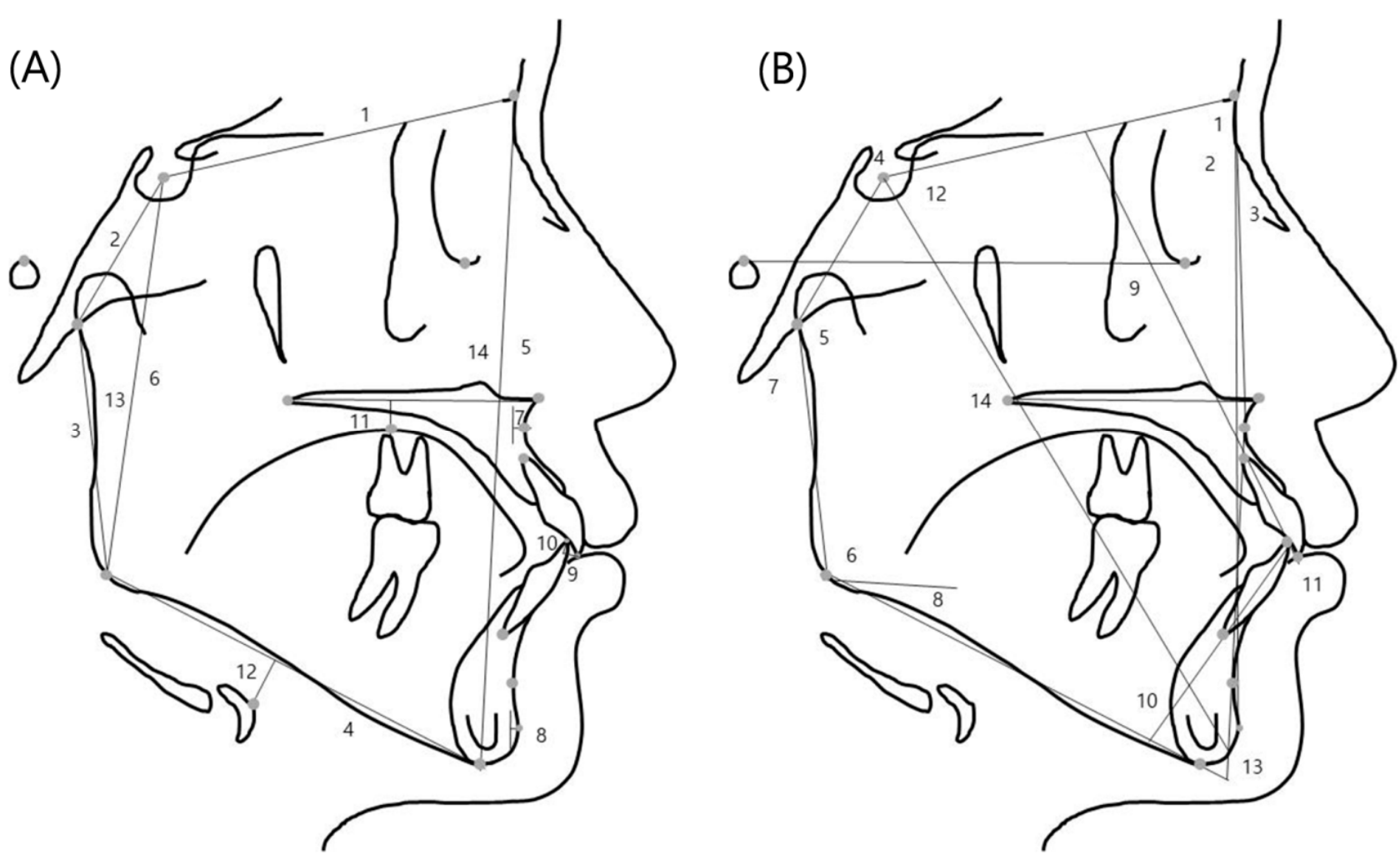

Figure 1. (A) Linear variables used in this study: 1, anterior cranial base (ACB); 2, posterior cranial base (PCB); 3, ramus height (RH); 4, mandibular body length (MBL); 5, anterior facial height (AFH); 6, posterior facial height (PFH); 7, point A to N perpendicular (A to N-perp); 8, pogonion to N perpendicular (Pog to N-perp); 9, overjet (OJ); 10, overbite (OB); 11 , dorsum of the tongue to palatal plane (Tp to PP); 12 , hyoidale to mandibular plane (H to MP); 13 , body to anterior cranial base ratio (body to ACB); 14, facial height ratio (FHR). (B) angular variables used in this study: 1, SNA angle; 2 , SNB angle; 3, ANB angle; 4, saddle angle; 5, articular angle; 6, gonial angle; 7, Björk sum; 8, Frankfort horizontal plane to mandibular plane angle (FMA); 9, upper incisor to FH angle (U1 to FH); 10, incisor mandibular plane angle (IMPA); 11, inter-incisor angle (IIA); 12, Y-axis to SN angle; 13, AB plane to mandibular plane angle (AB to MP); 14, palatal plane to Frankfort horizontal plane angle (PP to FH).

\subsection{Tongue and Lip Pressure Measurements}

Oral muscle strength measurements were performed using the Iowa Oral Performance Instrument (IOPI; Medical LLC, Carnation, WA, USA). The Iowa Oral Performance Instrument (IOPI) objectively measures the tongue and lip strength [19]. The IOPI measures the strength of the tongue by measuring the maximum pressure that an individual can produce in a standard-sized air-filled bulb by pressing the bulb against the roof of the mouth with the tongue. The peak pressure achieved is displayed on a liquid-crystal display (LCD). The units displayed are kilopascals $(\mathrm{kPa})$, based on the internationally recognized unit of pressure, the Pascal $(\mathrm{Pa})$. Currently, it is among the most commonly used measurement techniques available, and it has been validated in many previous studies [20,21].

The bulb was positioned longitudinally on the hard palate just posterior to the alveolar ridge to measure the anterior tongue pressure (TAP). Posterior tongue pressure (TPP) was measured with the bulb positioned more posteriorly, with the distal end of the bulb at the posterior border of the hard palate (Figure 2). After that, the bulb was positioned horizontally along the upper vestibule, which is between the upper lip and the gums and teeth to measure the lip pressure (LP) (Figure 3). 

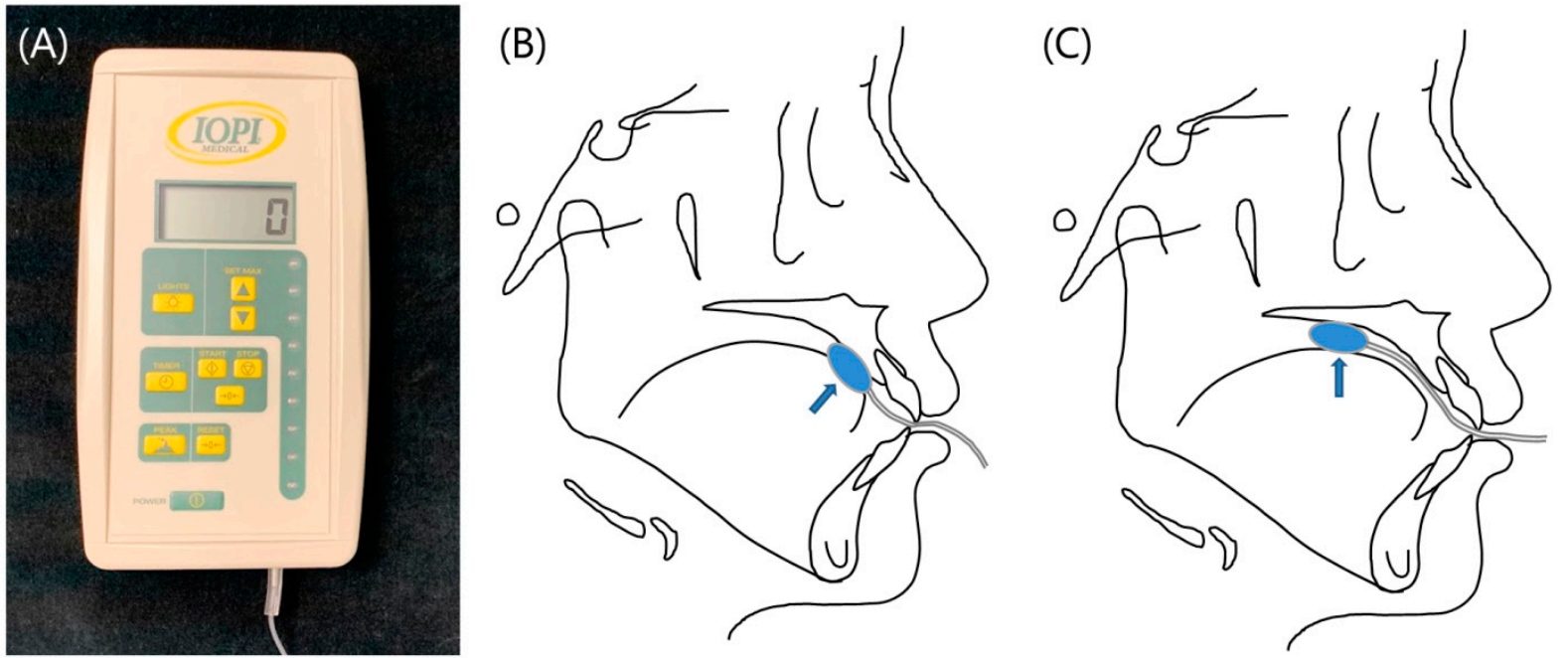

Figure 2. (A) Iowa Oral Performance Instrument (IOPI) device. (B) Position of the bulb (TAP, anterior tongue pressure). (C) Position of the bulb (TPP, posterior tongue pressure).
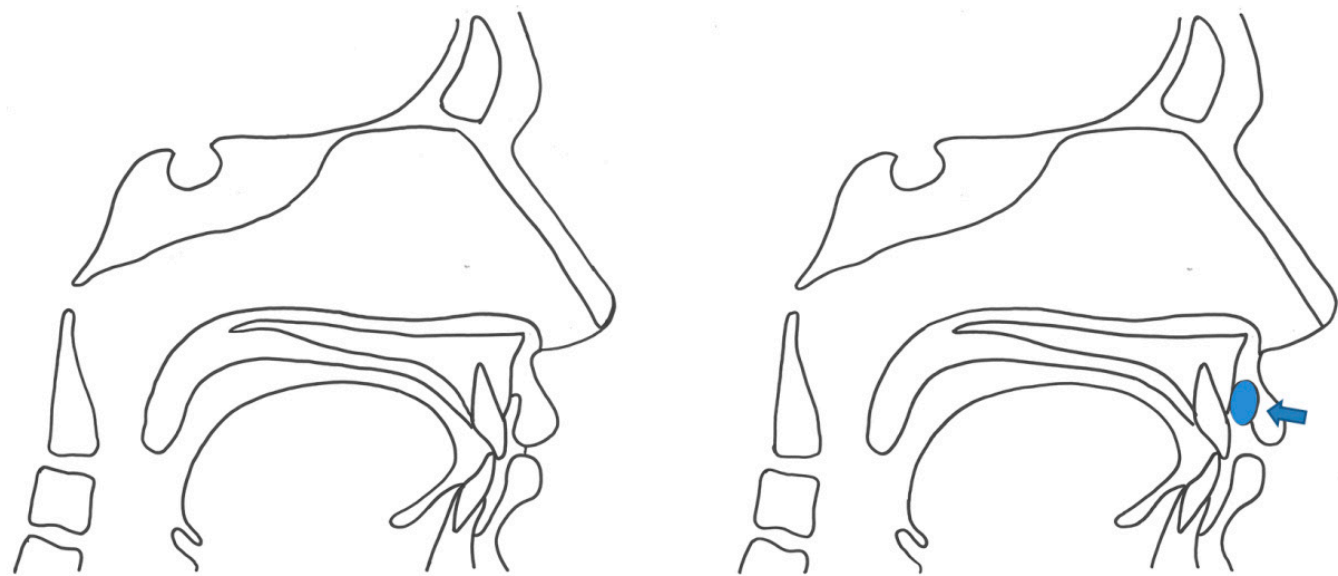

Figure 3. Position of the bulb (LP, lip pressure).

Patients were instructed to press the bulb as strongly as possible without biting the teeth and not to change the position of the bulb for each measurement. Three measurements were conducted for each patient at intervals of $30 \mathrm{~s}$, and the average value was used.

\subsection{Statistical Analysis}

To investigate whether the perioral muscle forces (TAP, TPP, and LP) were different according to sex, a t-test was performed. Pearson's correlation test was performed to analyse the correlation of cephalometric variables with perioral muscle forces. For each perioral muscle force (TAP, TPP, and LP), the upper/lower groups were divided based on the average value: $42 \mathrm{kPa}$ for anterior tongue pressure, $40 \mathrm{kPa}$ for posterior tongue pressure, and $24 \mathrm{kPa}$ for lip pressure (Table 1). Group comparisons between the high force group and low force group were performed using a $t$-test. Multiple linear regression analysis was used to establish the relationship between tongue and lip force and the cephalometric variables. The regression analysis was conducted three times by setting TAP, TPP, and LP as a response variable, respectively. Explanatory variables were age and 28 cephalometric measurements. The stepwise variable selection technique was used to reduce the number of explanatory variables and to select the significant ones. Statistical analyses were performed using SPSS software (ver 25.0; IBM Corp., Armonk, NY, USA). Statistical significance was accepted at $p<0.05$. 
Table 1. Sex and age distribution of patients with each group.

\begin{tabular}{cccccccc}
\hline & & \multicolumn{2}{c}{ TAP (kPa) } & \multicolumn{2}{c}{ TPP (kPa) } & \multicolumn{2}{c}{ LP (kPa) } \\
\cline { 3 - 8 } & & Low $(<\mathbf{4 2})$ & High $(\geq \mathbf{4 2 )}$ & Low $(<\mathbf{4 0 )}$ & High $(\geq \mathbf{4 0 )}$ & Low $(<\mathbf{2 4 )}$ & High $(\geq \mathbf{2 4})$ \\
\hline \multirow{2}{*}{ Sex } & Male & 45 & 59 & 46 & 58 & 57 & 47 \\
\multirow{2}{*}{ Age (y) } & Female & 55 & 35 & 57 & 33 & 62 & 28 \\
& Mean & 23.2 & 24.2 & 22.6 & 24.8 & 23.3 & 24.2 \\
& SD & 6.9 & 7.7 & 6.6 & 7.8 & 7.2 & 7.4 \\
\hline
\end{tabular}

$\mathrm{TAP}$, anterior tongue pressure; TPP, posterior tongue pressure; LP, Lip pressure.

\section{Results}

There was a statistically significant difference in tongue pressure between males and females $(p<0.05)$. Anterior and posterior tongue pressures were both higher in males (Figure 4). Meanwhile, no sex difference in lip pressure was observed.
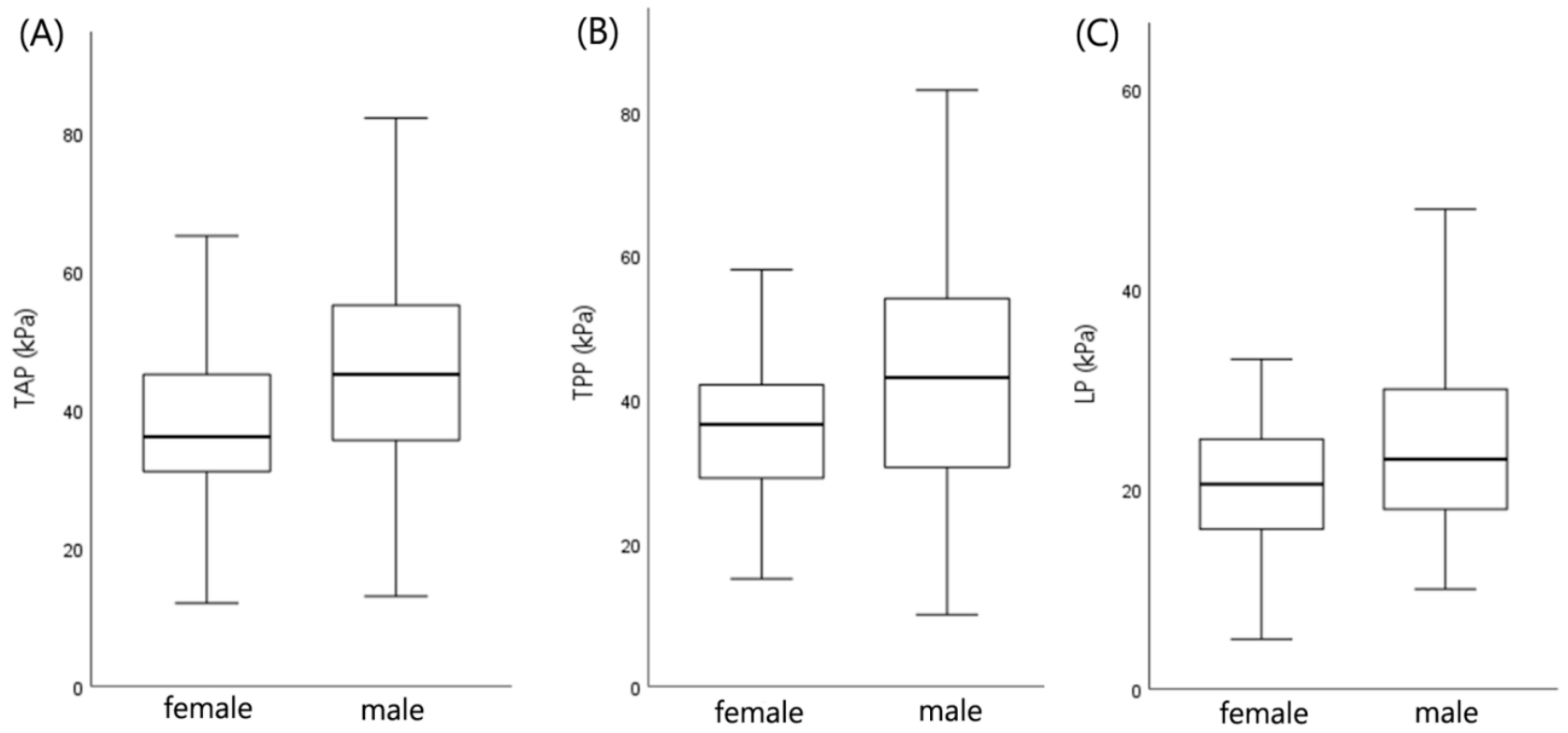

Figure 4. Sex-based pressure differences; (A) TAP, anterior tongue pressure. (B) TPP, posterior tongue pressure. (C) LP, lip pressure.

In the Pearson's correlation test, posterior cranial base (PCB) and posterior facial height (PFH) showed the highest positive correlation, and overjet (OJ) showed the highest negative correlation with anterior tongue pressure. Additionally, $\mathrm{PCB}$ and posterior facial height (PFH) showed the highest positive correlation with posterior tongue pressure. The angulation of the upper incisor to FH plane (U1 to FH) showed a negative correlation with lip pressure (Table 2).

Comparisons of the cephalometric measurements between the upper/lower groups are given in Tables 3-5. Variables that were different among groups were articular angle, PCB, OJ, and $\mathrm{H}$ to MP for anterior tongue pressure, and Björk sum, PCB, PFH, FHR, and ramus height for posterior tongue pressure. In the group with a low anterior tongue pressure, the articular angle and OJ were large, and the PCB and $\mathrm{H}$ to MP were short. In the group with a low posterior tongue pressure, the length of $\mathrm{PCB}, \mathrm{PFH}$, and ramus height were short, and the Björk sum and FHR were small.

In the case of lip pressure, OJ, IIA, and PCB showed significant differences between groups. The group with a low lip pressure tended to have a large OJ and small IIA and PCB. This shows that the pressure on the upper lip mainly affects the angle of the anterior teeth, not the skeletal-related measurements. However, the SDs for the OJ were high, showing a statistically significant difference, but showed high variability within the groups. 
Table 2. Correlation coefficients between cephalometric variables and perioral muscle force (TAP, TPP, LP) †.

\begin{tabular}{|c|c|c|c|c|c|c|}
\hline \multirow[b]{2}{*}{ Cephalometric Variable } & \multicolumn{2}{|c|}{ TAP } & \multicolumn{2}{|c|}{ TPP } & \multicolumn{2}{|c|}{ LP } \\
\hline & Correlation & $p$-Value & Correlation & $p$-Value & Correlation & $p$-Value \\
\hline $\mathrm{ACB}$ & 0.151 & $0.035 *$ & NS & NS & NS & NS \\
\hline PCB & 0.277 & $<0.001^{* * *}$ & 0.229 & $0.001 * *$ & NS & NS \\
\hline $\mathrm{RH}$ & 0.205 & $0.004^{* *}$ & 0.147 & $0.041 *$ & NS & NS \\
\hline PFH & 0.251 & $<0.001^{* * *}$ & 0.201 & $0.005 * *$ & NS & NS \\
\hline Pog to N-perp & 0.147 & $0.04 *$ & NS & NS & NS & NS \\
\hline $\mathrm{OJ}^{1}$ & -0.202 & $0.005^{* *}$ & NS & NS & NS & NS \\
\hline FHR & 0.183 & $0.011 *$ & 0.19 & $0.008^{* *}$ & NS & NS \\
\hline SNA & NS & NS & 0.149 & $0.038 *$ & NS & NS \\
\hline SNB & 0.161 & $0.025 *$ & 0.16 & $0.026 *$ & NS & NS \\
\hline ANB & -0.151 & $0.035 *$ & NS & NS & NS & NS \\
\hline Articular angle & -0.151 & $0.036^{*}$ & NS & NS & NS & NS \\
\hline Björk sum & -0.153 & 0.033 * & -0.174 & $0.015 *$ & NS & NS \\
\hline $\mathrm{U} 1$ to $\mathrm{FH}$ & NS & NS & NS & NS & -0.231 & $0.001 * *$ \\
\hline IIA & NS & NS & NS & NS & 0.211 & $0.003 * *$ \\
\hline $\mathrm{Y}$ axis to $\mathrm{SN}$ & -0.146 & $0.042 *$ & -0.146 & $0.042 *$ & NS & NS \\
\hline
\end{tabular}

† Only shows the variables for which a significant correlation was found.NS, not significant. TAP, anterior tongue pressure. TPP, posterior tongue pressure; LP, Lip pressure. ${ }^{*} p<0.05,{ }^{* *} p<0.01,{ }^{* * *} p<0.001$.

Table 3. Comparisons of cephalometric variables between TAP_low and TAP_high groups $(\mathrm{n}=194)$. *

\begin{tabular}{ccccc}
\hline Cephalometric Variables & TAP_Low & TAP_High & Significance & Comparisons \\
\hline Articular angle $\left(^{\circ}\right)$ & $148.2 \pm 8.0$ & $146.0 \pm 7.4$ & $0.022^{*}$ & TAP_low $>$ TAP_high \\
PCB $(\mathrm{mm})$ & $38.6 \pm 3.6$ & $39.7 \pm 4.2$ & $0.027^{*}$ & TAP_low $<$ TAP_high \\
OJ $(\mathrm{mm})$ & $2.5 \pm 4.0$ & $1.3 \pm 3.8$ & $0.016^{*}$ & TAP_low $>$ TAP_high \\
H to MP $(\mathrm{mm})$ & $13.0 \pm 5.6$ & $14.7 \pm 5.9$ & $0.019^{*}$ & TAP_low $<$ TAP_high \\
\hline
\end{tabular}

* Only shows the variables for which a statistically significant difference was found $(p<0.05)$. TAP_low, low anterior tongue pressure group; TAP_high, high anterior tongue pressure group. ${ }^{*} p<0.05$.

Table 4. Comparisons of cephalometric variables between TPP_low and TPP_high groups $(\mathrm{n}=194) .{ }^{*}$

\begin{tabular}{ccccc}
\hline Cephalometric Variables & TPP_Low & TPP_High & Significance & Comparisons \\
\hline Björk sum $\left(^{\circ}\right)$ & $398.2 \pm 7.4$ & $396.4 \pm 7.2$ & $0.045^{*}$ & TPP_low $>$ TPP_high \\
PCB $(\mathrm{mm})$ & $38.5 \pm 3.6$ & $39.8 \pm 4.3$ & $0.011^{*}$ & TPP_low $<$ TPP_high \\
PFH (mm) & $87.2 \pm 8.8$ & $90.2 \pm 9.0$ & $0.010^{*}$ & TPP_low $<$ TPP_high \\
FHR & $63.9 \pm 5.6$ & $65.6 \pm 5.9$ & $0.021^{*}$ & TPP_low $<$ TPP_high \\
RH $(\mathrm{mm})$ & $52.5 \pm 7.1$ & $54.5 \pm 7.7$ & $0.031^{*}$ & TPP_low $<$ TPP_high \\
\hline
\end{tabular}

* Only shows the variables for which a statistically significant difference was found $(p<0.05)$. TPP_low, low posterior tongue pressure group, TPP_high, high posterior tongue pressure group. ${ }^{*} p<0.05$.

Table 5. Comparisons of cephalometric variables between LP_low and LP_high groups $(\mathrm{n}=194)$.

\begin{tabular}{ccccc}
\hline Cephalometric Variables & LP_Low & LP_High & Significance & Comparisons \\
\hline IIA $\left(^{\circ}\right)$ & $125.3 \pm 11.9$ & $128.8 \pm 13.7$ & $0.034^{*}$ & LP_low $<$ LP_high \\
PCB $(\mathrm{mm})$ & $38.7 \pm 3.8$ & $39.7 \pm 4.1$ & $0.045^{*}$ & LP_low $<$ LP_high \\
OJ $(\mathrm{mm})$ & $2.4 \pm 3.9$ & $1.1 \pm 3.9$ & $0.013^{*}$ & LP_low $>$ LP_high \\
\hline
\end{tabular}

* Only shows the variables for which a statistically significant difference was found $(p<0.05)$. LP_low, low lip pressure group. LP_high, high lip pressure group. ${ }^{*} p<0.05$.

The results of the multiple linear regression analysis are summarised in Table 6. As shown in the table, anterior tongue pressure was significantly different according to the PCB and OJ. The regression coefficients indicated that an increase in anterior tongue pressure would be observed, as the PCB increased and OJ decreased. In contrast, a significant difference in posterior tongue pressure was observed according to PCB, Tp to PP, OJ, and age. The greater the distance from the palatal plane to the dorsum of tongue, the lower the posterior tongue pressure value. Regarding lip pressure, a decreasing tendency of the lip pressure was observed as the angle U1 to FH and FMA were increased. 
Table 6. Stepwise multiple regression analysis for the perioral muscle force.

\begin{tabular}{|c|c|c|c|c|c|c|}
\hline Dependent Variable & Statistically Significant Independent Variables & B & SE B & $p$-Value & Adjusted R-Quared & F-Statistic \\
\hline \multirow[t]{2}{*}{ TAP } & PCB (mm) & 0.86 & 0.24 & $<0.001 * * *$ & 0.091 & 10.62 \\
\hline & $\mathrm{OJ}(\mathrm{mm})$ & -0.55 & 0.25 & 0.027 * & & \\
\hline \multirow[t]{4}{*}{ TPP } & РCВ (mm) & 0.92 & 0.25 & $<0.001 * * *$ & 0.099 & 6.28 \\
\hline & $\mathrm{Tp}$ to $\mathrm{PP}(\mathrm{mm})$ & -0.56 & 0.21 & $0.01 *$ & & \\
\hline & $\mathrm{OJ}(\mathrm{mm})$ & -0.53 & 0.26 & $0.04 *$ & & \\
\hline & age & 0.29 & 0.13 & $0.03 *$ & & \\
\hline \multirow[t]{2}{*}{$\mathrm{LP}$} & $\mathrm{U} 1$ to $\mathrm{FH}\left({ }^{\circ}\right)$ & -0.48 & 0.11 & $<0.001 * * *$ & 0.095 & 11.14 \\
\hline & $\operatorname{FMA}\left({ }^{\circ}\right)$ & -0.45 & 0.14 & $0.001 * *$ & & \\
\hline
\end{tabular}

TAP, anterior tongue pressure; TPP, posterior tongue pressure; LP, Lip pressure; B, unstandardized regression coefficient; SE B, standard error of B. ${ }^{*} p<0.05,{ }^{* *} p<0.01, * * * p<0.001$.

Comparing the skeletal profilograms, it can be seen that the low TPP group showed characteristics of short ramus height and short PFH, and the low LP group showed a large OJ and small IIA, which indicates the proclination of the central incisors (Figure 5). In addition, the low TAP group exhibited characteristics of both groups (low TPP and low LP group).
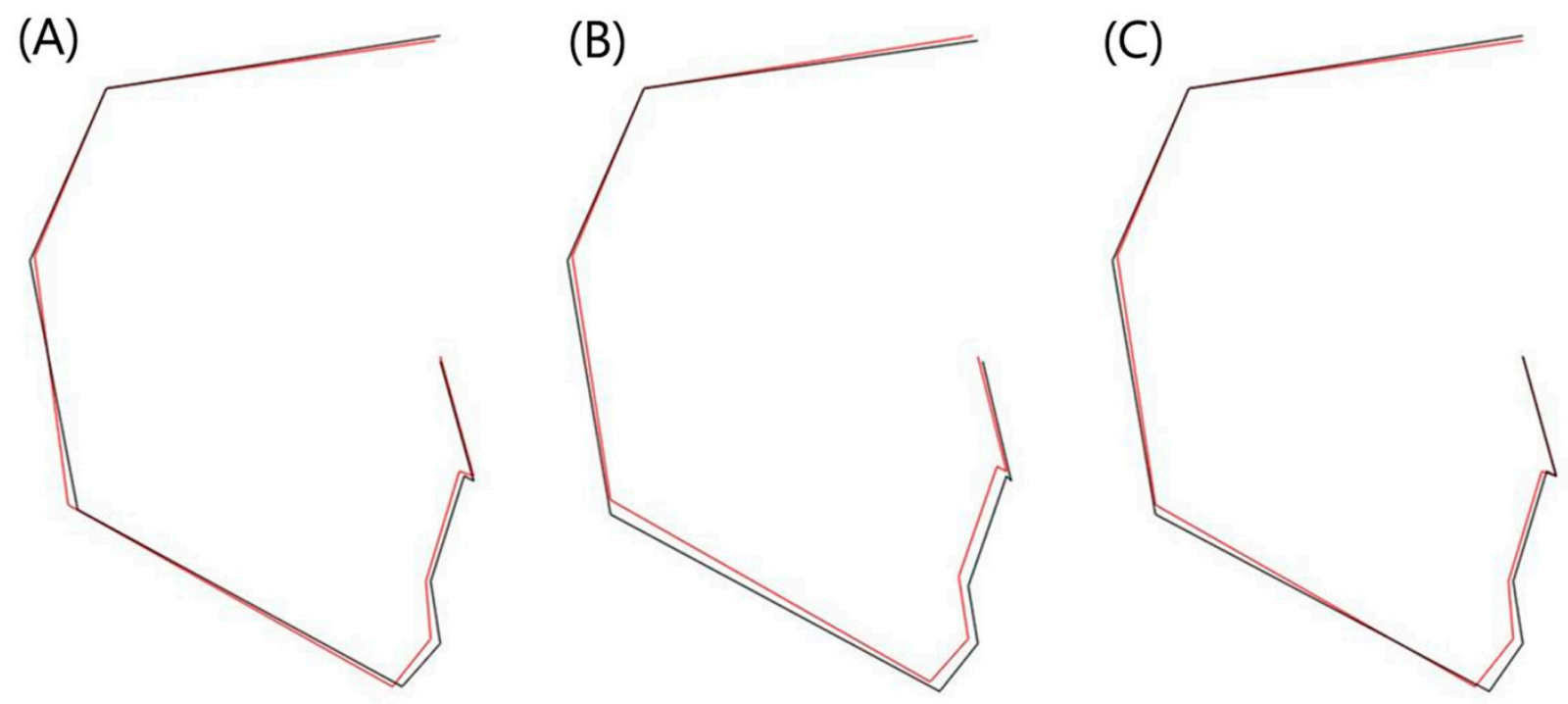

Figure 5. Comparison of skeletal profilograms between the high group (black line) and the low group (red line); (A) TAP, anterior tongue pressure. (B) TPP, posterior tongue pressure. (C) LP, lip pressure.

\section{Discussion}

Results obtained from previous studies regarding tongue pressure cannot be compared to one other because the measurement locations are different, and the measurement methods were not standardized. In this study, we used the Iowa Oral Performance Instrument (IOPI), an appropriate evaluation tool, to objectively measure the tongue strength [19]. In order to reduce the error when the force was not accurately applied during the first measurement, three measurements were performed, and the average value was used.

Anterior and posterior tongue pressures were both higher in males. As reported in previous studies, it is thought to be the result of basic muscle mass differences between men and women $[21,22]$. The study investigating the orofacial strength (tongue, lip, and buccinator muscle) of healthy adults showed that men had a greater strength than women in all measurements [22].

From the results of the correlation analysis of the perioral muscle force and cephalometric variables, anterior and posterior tongue pressure showed a positive association with PCB, RH, PFH, FHR, and SNB, and a negative association with Björk sum and Y-axis to $\mathrm{SN}$. According to these findings, patients with a vertical skeletal pattern who have a short ramus height and a clockwise-rotated mandible tend to have weak tongue strength. 
Variables related to the maxillary incisor angulation (U1 to FH and IIA) showed significant correlations with lip pressure. Dentofacial morphology according to the differences in the anterior tongue pressure showed a mixed tendency of dentofacial morphology according to the posterior tongue pressure and lip pressure. Anatomically, the upper and lower incisors are located between the tongue and the lips; therefore, if the anterior tongue pressure is strong and the lip pressure is weak, the maxillary anterior teeth are proclined, and the OJ becomes larger.

Through a comparative analysis between groups, skeletal pattern and skeletal size were found to be related to the tongue pressure. In particular, short posterior facial height and clockwise-rotated mandible were seen in the group with a low posterior tongue pressure. This is probably due to similar reasons, i.e., masticatory muscle strength tends to be low in the group with a skeletal open bite [23]. This shows that the pressure on the lips correlates with the angle of the anterior teeth.

In the multiple regression analysis between the perioral muscle force and the cephalometric variable, the statistically significant independent variables were $\mathrm{PCB}, \mathrm{Tp}$ to $\mathrm{PP}$, age, $\mathrm{OJ}, \mathrm{U} 1$ to $\mathrm{FH}$, and FMA. Anterior and posterior tongue pressure exhibited positive associations with PCB and negative associations with OJ. Lip pressure was found to be negatively associated with U1 to FH. Higher lip pressure results in the inclination of the upper incisors lingually. Our finding was consistent with that of a previous study in which subjects with Angle Class II division 1 relationships showed a lower lip-closing force than those with Angle Class I relationships [2].

The limitation of this study was that only upper lip pressure was evaluated, and the role of the lower lip pressure remained unknown. Another limitation was that only two functional parameters of the tongue were examined (TAP and TPP), whereas there was no assessment of the same parameters at rest. This could also be a suggestion for future studies. Tongue posture and function have been associated with the aetiology of malocclusions and posttreatment stability. Smithpeter demonstrated that combined myofunctional therapy has shown better results in long-term maintenance in anterior open bite closure than orthodontic treatment alone [24]. Meazzini et al. reported that the presence or absence of a tongue with glossectomy did not affect mandibular growth in patients with BeckwithWiedermann syndrome [25]. Once the effects of orofacial myofunctional therapy (OMT) on the tongue strength have been identified, they will be used as scientific evidence to support the importance of OMT. The treatment of malocclusion could probably benefit from the establishment of harmonious relationships between the perioral muscles. It is also necessary to study how perioral muscles affect the growth pattern in the future.

\section{Conclusions}

Anterior and posterior tongue pressures were higher in males than in females. The group with a low posterior tongue pressure had a short posterior facial height, short ramus height, and clockwise-rotated mandible. Lip pressure affects the angle of the anterior teeth, not the skeletal-related measurements. Higher lip pressure results in the inclination of the upper incisors lingually. Anterior tongue pressure showed a mixed tendency of posterior tongue pressure and lip pressure. The group with a low anterior tongue pressure had a large $\mathrm{OJ}$ and short posterior cranial base length.

Author Contributions: Conceptualization, Y.-S.L. and T.-W.K.; methodology, J.R. and S.-H.B.; software, J.R. and I.-H.Y.; validation, W.H.L. and S.-K.J.; formal analysis, Y.-S.L.; investigation, Y.S.L. and J.R.; resources, T.-W.K.; data curation, S.-K.J.; writing-original draft preparation, Y.-S.L.; writing-review and editing, S.-K.J.; visualization, I.-H.Y.; supervision, S.-H.B.; project administration, W.H.L.; funding acquisition, T.-W.K. All authors have read and agreed to the published version of the manuscript.

Funding: This research was funded by Seoul National University Dental Hospital Research Fund, grant number 05-2019-0019. 
Institutional Review Board Statement: The study was conducted according to the guidelines of the Declaration of Helsinki and approved by the Institutional Review Board of Seoul National University Dental Hospital (CRI18001, 16/03/2019).

Informed Consent Statement: Informed consent was obtained from all subjects involved in the study.

Data Availability Statement: The data underlying this article will be shared on reasonable request from the corresponding author.

Conflicts of Interest: The authors declare no conflict of interest.

\section{References}

1. Brodie, A.G. Consideration of musculature in diagnosis, treatment, and retention. Am. J. Orthod. 1952, 38, 823-835. [CrossRef]

2. Lambrechts, H.; De Baets, E.; Fieuws, S.; Willems, G. Lip and tongue pressure in orthodontic patients. Eur. J. Orthod. 2010, 32, 466-471. [CrossRef] [PubMed]

3. Primozic, J.; Farčnik, F.; Perinetti, G.; Richmond, S.; Ovsenik, M. The association of tongue posture with the dentoalveolar maxillary and mandibular morphology in Class III malocclusion: A controlled study. Eur. J. Orthod. 2012, 35, 388-393. [CrossRef] [PubMed]

4. Weinstein, S.; Haack, D.C.; Morris, L.Y.; Snyder, B.B.; Attaway, H.E. On an equilibrium theory of tooth position. Angle Orthod. 1963, 33, 1-26.

5. Jung, M.-H.; Yang, W.-S.; Nahm, D.-S. Maximum Closing Force of Mentolabial Muscles and Type of Malocclusion. Angle Orthod. 2010, 80, 72-79. [CrossRef] [PubMed]

6. Kuroda, T.; Ono, T. Diagnosis and management of oral dysfunction. World J. Orthod. 2000, 1, 125-133.

7. Fröhlich, K.; Ingervall, B.; Thüer, U.; Hlich, K.F.; Er, U.T. Further studies of the pressure from the tongue on the teeth in young adults. Eur. J. Orthod. 1992, 14, 229-239. [CrossRef] [PubMed]

8. Ackerman, J.L.; Proffit, W.R. Soft tissue limitations in orthodontics: Treatment planning guidelines. Angle Orthod. 1997, $67,327-336$.

9. Ruan, W.-H.; Chen, M.-D.; Gu, Z.-Y.; Lu, Y.; Su, J.-M.; Guo, Q. Muscular forces exerted on the normal deciduous dentition. Angle Orthod. 2005, 75, 785-790.

10. Adesina, B.A.; Otuyemi, O.D.; Kolawole, K.A.; Adeyemi, A.T. Assessment of the impact of tongue size in patients with bi-maxillary protrusion. Int. Orthod. 2013, 11, 221-232.

11. Proffit, W.R. Equilibrium theory revisited: Factors influencing position of the teeth. Angle Orthod. 1978, 48, 175-186.

12. Kurabeishi, H.; Tatsuo, R.; Makoto, N.; Kazunori, F. Relationship between tongue pressure and maxillofacial morphology in Japanese children based on skeletal classification. J. Oral Rehabil. 2018, 45, 684-691. [CrossRef]

13. Doto, N.; Yamada, K. The relationship between maximum lip closing force and tongue pressure according to lateral craniofacial morphology. Orthod. Waves 2015, 74, 69-75. [CrossRef]

14. Posen, A.L. The influence of maximum perioral and tongue force on the incisor teeth. Angle Orthod. 1972, 42, 285-309. [PubMed]

15. Azevedo, N.D.; Lima, J.C.; Furlan, R.M.M.M.; Motta, A.R. Tongue pressure measurement in children with mouth-breathing behaviour. J. Oral Rehabil. 2018, 45, 612-617. [CrossRef] [PubMed]

16. Ruan, W.-H.; Su, J.-M.; Ye, X.-W. Pressure from the lips and the tongue in children with class III malocclusion. J. Zhejiang Univ. Sci. B 2007, 8, 296-301. [CrossRef]

17. Posen, A.L. The application of quantitative perioral assessment to orthodontic case analysis and treatment planning. Angle Orthod. 1976, 46, 118-143.

18. Jung, M.-H.; Yang, W.-S.; Nahm, D.-S. Effects of upper lip closing force on craniofacial structures. Am. J. Orthod. Dentofac. Orthop. 2003, 123, 58-63. [CrossRef]

19. Adams, V.; Mathisen, B.; Baines, S.; Lazarus, C.; Callister, R. A Systematic Review and Meta-analysis of Measurements of Tongue and Hand Strength and Endurance Using the Iowa Oral Performance Instrument (IOPI). Dysphagia 2013, 28, 350-369. [CrossRef]

20. Youmans, S.R.; Stierwalt, J.A.G. Measures of Tongue Function Related to Normal Swallowing. Dysphagia 2006, $21,102-111$. [CrossRef] [PubMed]

21. Youmans, S.R.; Youmans, G.L.; Stierwalt, J.A.G. Differences in Tongue Strength Across Age and Gender: Is There a Diminished Strength Reserve? Dysphagia 2008, 24, 57-65. [CrossRef]

22. Park, J.-S.; You, S.-J.; Jeong, C.H. Age and Sex Differences in Orofacial Strength of Healthy Korean Adult. Korean J. Occup. Ther. 2013, 21, 103-116.

23. Ingervall, B.; Thüer, U.; Kuster, R. Lack of correlation between mouth-breathing and bite force. Eur. J. Orthod. 1989, 11, 43-46. [CrossRef]

24. Smithpeter, J.; Covell, D. Relapse of anterior open bites treated with orthodontic appliances with and without orofacial myofunctional therapy. Am. J. Orthod. Dentofac. Orthop. 2010, 137, 605-614. [CrossRef] [PubMed]

25. Meazzini, M.C.; Besana, M.; Tortora, C.; Cohen, N.; Rezzonico, A.; Ferrari, M.; Autelitano, L. Long-term longitudinal evalutation of mandibular growth in patients with Beckwith-Wiedemann Syndrome treated and not treated with glossectomy. J. Craniomaxillofac. Surg. 2020, 48, 1126-1131. [CrossRef] [PubMed] 